\title{
LRIG1 combined with cisplatin enhances bladder cancer lesions via a novel pathway
}

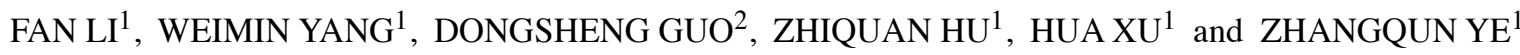 \\ Departments of ${ }^{1}$ Urology and ${ }^{2}$ Neurosurgery, Tongji Hospital, Tongji Medical College, \\ Huazhong University of Science and Technology, Wuhan, Hubei 430030, P.R. China
}

Received November 22, 2010; Accepted February 2, 2011

DOI: 10.3892/or.2011.1227

\begin{abstract}
One aspect of chemotherapy insensitivity and resistance results from induction of epidermal growth factor receptor (EGFR) internalization and initial DNA damage repair in response to DNA-damaging stimuli, such as cisplatin (CDDP). Previously, we found that leucine-rich repeats and immunoglobulin-like domains 1 (LRIG1), as one of the natural ligands of EGFR, could combine with and downregulate the expression of EGFR in bladder cancer cells. This finding interested us and we hypothesized that LRIG1 could be a novel candidate for facilitating cisplatin-induced bladder cancer cell lesions. To investigate this further, we overexpressed LRIG1 with an adenovirus vector in EJ/T24 bladder cancer cells and investigated total EGFR, nuclear expression of phosphorylated EGFR (pEGFR) and cell lesions with exposure to CDDP. CDDP-induced nuclear pEGFR levels accumulated with time and were decreased by LRIG1 overexpression. LRIG1-transduced cells treated with CDDP had more severe DNA damage, cellular apoptosis, growth inhibition and reversal of invasion. These preclinical studies indicate that LRIG1 may represent a new therapeutic approach to improve the response of bladder cancer to chemotherapy through a novel pathway.
\end{abstract}

Correspondence to: Dr Zhangqun Ye, Department of Urology, Tongji Hospital, Tongji Medical College, Huazhong University of Science and Technology, Wuhan, Hubei 430030, P.R. China

E-mail: zhangqun_ye@yahoo.com.cn

Abbreviations: BSA, bovine serum albumin; CASP, comet assay software project; CDDP, cisplatin; DAB, diaminobenzidine; DMEM, Dulbecco's modified Eagle's medium; DMSO, dimethylsulfoxide; DNA-PK, DNA-dependent kinase; EGFR, epidermal growth factor receptor; FACS, fluorescence activated cell sorting; FBS, fetal bovine serum; FCM, flow cytometry; GFP, green fluorescence protein; LRIG1, leucine-rich repeats and immunoglobulin-like domains 1; MOI, multiplicity of infection; PCNA, proliferation cells nuclear antigen; pEGFR, phosphorylation EGFR; PI, propidium iodide; RT-PCR, reverse-transcription polymerase chain reaction; SCGE, single-cell gel electrophoresis

Key words: epidermal growth factor receptor, nuclear import, LRIG1, chemotherapy, DNA damage, bladder cancer

\section{Introduction}

Seventy percent of bladder cancer is superficial and can be managed by transurethral resection of the bladder and/or the instillation of intravesical chemotherapeutic agents. However, the residual $20-30 \%$ of tumors with muscle invasion and 5\% with clinically evident distant metastasis usually require chemotherapy to enhance survival rate and prolong life span (1). Chemotherapy drugs that induce DNA damage, such as by cross-linking or causing single/double-strand breaks, are classic and important components of chemotherapy. Cisplatin (CDDP) is one of the most frequently adopted DNA-damaging agents and results in response rates of $60-80 \%$ in patients with advanced bladder cancer. As a result of drug insensitivity and/or resistance, however, CDDP-treatment only leads to complete remission in $15 \%$ of advanced bladder cancer patients (2). Insensitivity and/or resistance to CDDP could be due to reduced drug accumulation, increased nuclear DNA repair and detoxification of agents in the cellular cytoplasm (3). Thus, enhancing the effectiveness of DNA-damaging drugs and reducing their side effects could have beneficial effects for the treatment of bladder cancer.

Exposure to a variety of DNA-damaging stimuli, such as ionizing radiation, UV-radiation, hypoxia and oxidative stress, induces internalization of EGFR non-ligands from the membrane into the nucleus (4-7). As a consequence of the nuclear import of EGFR, DNA-dependent kinase (DNA-PK) is up-regulated and promotes the repair of damaged DNA (5). These findings suggest that the limitations of DNA-damaging drugs are caused, at least partly, by nuclear import of EGFR and DNA repair. Theoretically, inhibition of EGFR translocation could enhance the effectiveness of DNA-damaging therapies and delay resistance. Blocking the nuclear import of EGFR can be achieved by targeting EGFR and EGFR-specific tyrosine kinase. These strategies have been shown, in preclinical and clinical trials, to sensitize patients to ionizing radiation therapy $(6,7)$. To our knowledge, however, no research has been conducted on EGFR translocation and its value on chemotherapy in bladder cancer.

Leucine-rich repeats and immunoglobulin-like domains 1 (LRIG1) is a transmembrane leucine-rich repeat and immunoglobulin (Ig)-like domain-containing protein and is a newly identified negative regulator of the ErbB family of receptor 
tyrosine kinases (8). The inactivation of LRIG1 in rodents promotes epidermal cell hyperplasia, suggesting involvement in EGFR regulation. Previous work has demonstrated up-regulation of the LRIG1 transcript and protein upon stimulation with EGF as well as physical association of the encoded protein with the four EGFR orthologs in mammals. Up-regulation of LRIG1 is followed by enhanced ubiquitylation and degradation of EGFR. Previously, we found that LRIG1, as one of the natural ligands of EGFR that were absent or decreased in malignant bladder tissue compared to normal bladder tissue, could combine with and down-regulate the expression of EGFR. Up-regulation of LRIG1 expression by plasmid transfection in the bladder cancer cell line, BIU-87 (superficial), resulted in inhibition of cell proliferation and attenuation of cell metastasis and invasion, demonstrating that LRIG1 is a tumor suppression gene (TSG) (9). These findings indicated that LRIG1 could be a novel candidate for down-regulating CDDP-induced nuclear import of EGFR.

In this study, we have investigated the expression levels of activated EGFR (phosphorylated EGFR, pEGFR Tyr 845 ) in EJ/T24 bladder cancer cell nuclei, after exposure to CDDP. Moreover, we up-regulated the expression of LRIG1 by adenovirus vector and investigated whether nuclear pEGFR could be down-regulated. Furthermore, we studied the possible mechanism of this phenomenon and whether CDDP-induced DNA damage could be intensified.

\section{Materials and methods}

Cell line, cell culture and reagents. The human bladder cancer cell line, EJ/T24, was kindly provided by Dr Zhulin Liang. Cells were maintained at $37^{\circ} \mathrm{C}$ in a humidified atmosphere of $5 \% \mathrm{CO}_{2}$ in high glucose Dulbecco's modified Eagle's medium (DMEM) supplemented with $10 \%$ (v/v) fetal bovine serum (FBS) (Gibco-BRL, Gaithersburg, MD, USA). The medium was replaced every 3 days. Cells were checked routinely and passaged every 5 or 6 days. CDDP was dissolved in DMEM to $1 \mu \mathrm{g} / \mathrm{ml}$. MG132 from Sigma was dissolved in dimethylsulfoxide (DMSO) (final concentration $<5 \%$ ) and adjusted to $5 \mu \mathrm{M}$.

Construction of the adenoviral vector encoding human LRIG1. The pLRIG1-GFP plasmid containing full-length human LRIG1 cDNA was kindly provided by Professor Håkan Hedman and Dr Jonas Nilsson (Umeå University Hospital, Sweden). Human LRIG1 cDNA was amplified from the pLRIG1-GFP plasmid by PCR. Both the forward (5'-GTA GAA CGC AGA TCGAATTCA TGG CGC GGC CGG TC-3') and the reverse (5'-CCC TTG CTC ACC ATGAATTCG CTT TTT GGT GCC AAC AGC-3') primers contained an EcoRI site (underlined). The PCR products were digested and inserted into the EcoRI site of the shuttle plasmid pDC315GFP (Genechem Gene Technology Company Ltd., Shanghai, China) to yield pDC315-GFP-LRIG1. The pDC315-GFPLRIG1 sequence was verified by PCR using the primers described above. HEK-293 cells were then cotransfected with pDC315-GFP-LRIG1 and the helper plasmid pBHGlox (delta) E1, 3 Cre using Lipofectamine 2000 transfection reagent. Recombinant adenovirus encoding human LRIG1 was constructed using AdMax Kit D (Microbix Biosystems Inc.,
Canada). Transfected cells were collected when typical CPE appeared for all cells and over 50\% of cells had detached from the flask. The cells were then washed with PBS and subjected to freeze/thaw at $-70 / 37^{\circ} \mathrm{C}$ three times. The cell lysates were then centrifuged at $3000 \mathrm{rpm}$ at $4^{\circ} \mathrm{C}$ for $10 \mathrm{~min}$, and the supernatant was collected. This supernatant underwent three rounds of in vitro amplification, two rounds of purification by density gradient centrifugation and dialysis. After four passages, the titer of the rAd-LRIG1 was calculated using an endpoint dilution assay. Western blotting was used to evaluate whether the rAd-LRIG1 had been generated successfully. rAd-GFP, a recombinant adenovirus carrying the GFP gene, was produced in addition to a control vector.

Semi-quantitative RT-PCR. Total RNA was isolated with TRIzol Reagent (Invitrogen, USA). After the amount of total RNA was determined using ultraviolet spectrophotometry, the first strand of cDNA was synthesized from $2 \mu \mathrm{g}$ of total RNA using M-MLV, Oligo-dT18 primer and ReveTra Ace. For semi-quantitative PCR analysis, cDNA was amplified by Taq DNA polymerase. The human actin gene was used as an internal control. Sense, (5'-TGA TGG TCT GTC ACG GTCG3') and antisense (5'-TTG CTG ATG TTG TTT CGC TG-3') DNA primer sequences for human LRIG1 were designed. The amplicon size was $198 \mathrm{bp}$. PCR amplified products were separated by electrophoresis on $1.5 \%$ agarose gels and visualized by staining with $0.5 \%$ Goldview. Then, gel images were obtained.

Western blot analysis. Cells were washed with 0.01 M PBS once. Total proteins were extracted from cell lysates using Mammalian Protein Extraction Reagent (M-PER). Nuclear protein extracts were prepared according to the instructions of the NE-PER Nuclear and Cytoplasmic Extraction Kit (Pierce, IL, USA). The protein concentration of each sample was measured with micro-BCA protein assay reagent (Pierce Chemical Co., USA). Protein samples were added into loading buffer and denatured at $100^{\circ} \mathrm{C}$ for $5 \mathrm{~min}$. Samples were subjected to electrophoresis in an $8 \%$ SDS-polyacrylamide gel and transferred to a nitrocellulose membrane. TBST buffer containing 5\% non-fat dry milk was used to block nonspecific binding for $90 \mathrm{~min}$ at room temperature. Primary antibodies directed against LRIG1 (goat anti-human IgG, 1:300 dilution), $\beta$-actin (rabbit anti-human $\operatorname{IgG}, 1: 600$ dilution), EGFR (mouse monoclonal IgG, 1:200 dilution) and phospho-EGFR Tyr 845 (polyclonal IgG, 1:300 dilution) were incubated overnight at $4^{\circ} \mathrm{C}$ (Santa Cruz). Samples were then washed three times with TBST, and secondary antibodies (rabbit anti-goat IgG, 1:3000 dilution; goat anti-mouse IgG, 1:2000 dilution; goat anti-rabbit, 1:3000 dilution, Santa Cruz) were applied at $37^{\circ} \mathrm{C}$ for $90 \mathrm{~min}$. Samples were again washed three times with TBST. The blots were visualized by enhanced chemiluminescence color reagent kit (Pierce) and quantified by densitometry.

Single-cell gel electrophoresis (SCGE). Cells were treated and washed with 0.1 M PBS before trypsinization. SCGE was carried out in accordance with the standard procedure (10). Twenty-five microliters $\left(1 \times 10^{7} / \mathrm{ml}\right)$ of cells was mixed with $75 \mu \mathrm{l}$ of $0.5 \%$ low-melting-point agarose (Fisher Scientific) 
and were spread on a frosted microscope slide that had been pre-coated with normal melting point agarose, and coagulated at $4^{\circ} \mathrm{C}$ for at least $15 \mathrm{~min}$. Slides were placed in cold lysis solution for at least $1 \mathrm{~h}$. After lysis, the slides were washed three times for $5 \mathrm{~min}$ each in PBS. Slides were then incubated for $60 \mathrm{~min}$ in alkaline unwinding buffer $(\mathrm{pH}>13)$ in the dark at $4^{\circ} \mathrm{C}$ and were electrophoresed under the neutral conditions for $20 \mathrm{~min}$ at $20 \mathrm{~V}$ and $200 \mathrm{~mA}$. Slides were neutralized in $0.4 \mathrm{M}$ Tris, $\mathrm{pH} 7.5$, dried and stained for analysis with propidium iodide (PI; $50 \mu \mathrm{g} / \mathrm{ml}$, Sigma, USA) for at least $15 \mathrm{~min}$. Images of at least 50 cells per sample were captured using a fluorescence microscope. Individual images were evaluated by using Comet Assay Software Project Image software, and the mean of the Olive tail moment was calculated as a measure of DNA damage (11).

Cell cycle analysis by FCM. Different phases of the cell cycle are characterized by differing DNA content. PI is a fluorescent dye that binds strongly to DNA at a ratio of 1:1, hence varying PI intensity reflects the different DNA content of each phase of the cell cycle. Synchronization was achieved by serum starving cells, and then cells were treated as in Fig. 6. Cells were then harvested with trypsin-EDTA, washed twice with chilled PBS and fixed with $70 \%$ ethanol at $20^{\circ} \mathrm{C}$ overnight. The fixed cells were pelleted, re-suspended in PI/ RNase/PBS (PI, $100 \mu \mathrm{g} / \mathrm{ml}$ and RNase A, $10 \mu \mathrm{g} / \mathrm{ml}$ ) for at least $30 \mathrm{~min}$ at room temperature in the dark, then filtered through a nylon mesh of 400 screen meshes. At least $1 \times 10^{5}$ cells were analyzed by a fluorescence-activated cell sorting (FACS) caliber II sorter and CellQuest FACS system (BD Biosciences, USA). This experiment was repeated three times. The percentage of cells in $G_{0} / G_{1}, S$ and $G_{2} / M$ phases was determined by FACSCalibur.

Annexin V-APC/7-AAD double labeling for FCM-assessed apoptosis. The Annexin V-APC (Bender Medsystems, Vienna, Austria) was used to detect apoptosis according to the manufacturer's instructions. Synchronization was achieved by serum starving cells for $24 \mathrm{~h}$. Cells were then harvested by trypsinization and washed twice with cold PBS (0.1 M, pH 7.2). The cells were centrifuged at $2000 \mathrm{rpm}$ for $5 \mathrm{~min}$, then the supernatant was discarded and the pellet was re-suspended in $1 \mathrm{X}$ binding buffer at a density of $1 \times 10^{6}$ cells $/ \mathrm{ml}$. Each suspension $(100 \mu \mathrm{l})$ was transferred into individually labeled tubes and incubated with $5 \mu \mathrm{l}$ of APC-conjugated Annexin V and $5 \mu \mathrm{l}$ of 7-AAD (Keygene Co., Nanjing, China) for $15 \mathrm{~min}$ at room temperature in the dark. PBS binding buffer $(500 \mu \mathrm{l})$ was added to each sample tube without washing and analyzed within $1 \mathrm{~h}$ by FACS using CellQuest Research Software (BD Biosciences). Each group was measured three times.

Immunohistochemical staining. Cultured cells grown on cover slides were fixed with $4 \%$ paraformaldehyde for $30 \mathrm{~min}$ and permeabilized for $20 \mathrm{~min}$ with $0.1 \%$ Triton $\mathrm{X}-100$. Endogenous peroxidase activity was quenched with $3 \%$ hydrogen peroxidase in methanol for 30 min followed by $5 \%$ bovine serum albumin (BSA) in PBS for $30 \mathrm{~min}$. Cells were incubated for $1 \mathrm{~h}$ with monoclonal mouse anti-human proliferating cell nuclear antigen (PCNA, PC10, Cell Signaling
Technology, USA) diluted 1:4000 in PBS containing 3\% BSA. Negative controls were incubated with PBS instead of the primary antibody. Secondary antibodies were conjugated with HRP. All incubations were conducted at room temperature. Slides were rinsed three times for $10 \mathrm{~min}$ each in PBST between each step. Diaminobenzidine (DAB) was used as the chromogen. The slides were rinsed with PBS buffer, $\mathrm{pH} 7.6$ in $\mathrm{NaCl}$ solution, counterstained with Harris hematoxylin for 5 min, rinsed in water, differentiated, dehydrated with graded alcohols, bathed in fresh xylene and covered with gummi. The cells were evaluated by microscopy. PCNA expression was evaluated according to the percentage of stained cells and the intensity of staining.

Matrigel invasion assay. Motility and invasion capability in vitro were measured by a transwell chamber assay. Diluted Matrigel solution $(100 \mu \mathrm{l})$ was put into the upper chamber of the transwell inserts $(6.5 \mathrm{~mm}, 8 \mu \mathrm{m}$ pore size, Costar Inc., USA). Then, inserts were incubated at $37^{\circ} \mathrm{C}$ overnight to allow the gelling of the Matrigel and were then pretreated with serum-free DMEM medium at $37^{\circ} \mathrm{C}$ for $1 \mathrm{~h}$. Cells were seeded at a density of $1 \times 10^{5}$ per well in $100 \mu$ DMEM medium without FCS. The lower chambers of the transwell were filled with $600 \mu 1$ DMEM containing $10 \%$ FCS. The transwell inserts were then incubated at $37^{\circ} \mathrm{C}$ in $5 \% \mathrm{CO}_{2}$ for $24 \mathrm{~h}$ to allow cells to migrate. At the end of the incubation, the cells on the upper side of the insert filter were completely removed by wiping with a cotton swab. Cells that had migrated through the Matrigel-coated filter were fixed in $4 \%$ paraformaldehyde for $15 \mathrm{~min}$ and stained with $0.1 \%$ hexamethylpararosaniline for $20 \mathrm{~min}$. Cells that had invaded the Matrigel and reached the lower surface of the filter were counted under a light microscope. The assay was performed in triplicate.

Statistical analysis. Results are expressed as the means \pm standard deviation. Statistical analyses were performed using SPSS 13.0 statistical software (SPSS Inc., Chicago, IL). ANOVA was used to compare means of multiple groups. The S-N-K method was used for paired comparison. Significance was defined as $\mathrm{p}<0.05$ and $\mathrm{p}<0.01$.

\section{Results}

CDDP induced nuclear import of EGFR in EJ/T24 cells. Expression of pEGFR (Tyr 845) in EJ/T24 cell nuclei rose within $10 \mathrm{~min}$ of CDDP $(1 \mu \mathrm{g} / \mathrm{ml})$ treatment. Nuclear pEGFR levels in EJ/T24 cells increased with time and reached a peak of approximately 3 -fold compared to baseline levels $(\mathrm{p}<0.05)$ at $30 \mathrm{~min}$ (Fig. 1). However, changes in EGFR expression levels in EJ/T24 cells among four different time points were not significant $(\mathrm{p}>0.05)$. These results indicate that, at least at the time points measured, CDDP did not regulate EGFR expression in the immediate early phase of DNA damage, and the CDDP-induced accumulation of nuclear pEGFR was not caused by increased EGFR expression.

Ad-LRIG1 transduction inhibited EGFR nuclear import by down-regulating EGFR expression. Preliminary experiments were used to determine the multiplicity of infection (MOI) 


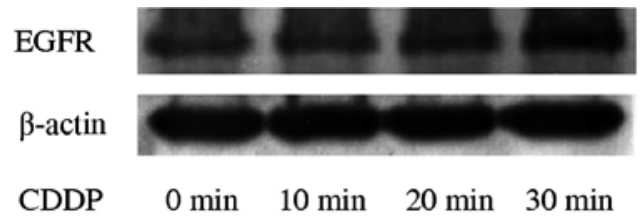

A

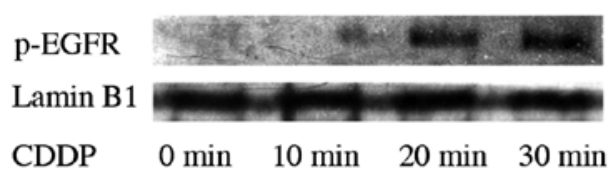

B

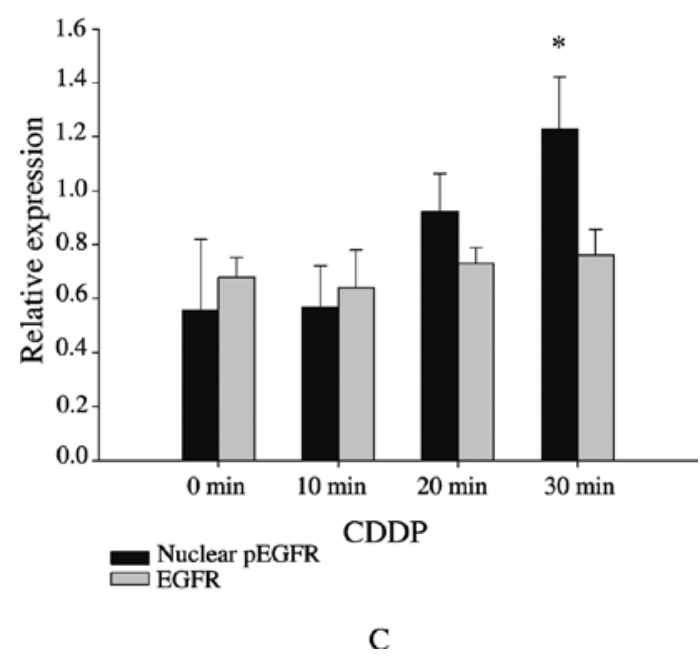

Figure 1. The expression of EGFR and nuclear pEGFR after CDDP treatment in the EJ/T24 cell line. (A) EGFR expression levels in cells treated with CDDP at $1 \mu \mathrm{g} / \mathrm{ml}$ for 10,20 and $30 \mathrm{~min}$; (B) nuclear pEGFR (Tyr 845) expression levels in cells treated with CDDP at $1 \mu \mathrm{g} / \mathrm{ml}$ for 10,20 and $30 \mathrm{~min}$; (C) relative expression of EGFR and nuclear pEGFR (Tyr 845) in cells treated with CDDP at $1 \mu \mathrm{g} / \mathrm{ml}$ for 10,20 and $30 \mathrm{~min}(" \mathrm{p}<0.05)$.

(16-25:1) for infection of EJ/T24 cells with the adenovirus vector containing LRIG1 (Ad-LRIG1). EJ/T24 cells were then infected as described. Total RNA and protein were extracted and analyzed by reverse transcription polymerase chain reaction (RT-PCR) and Western blotting, respectively. Western blotting and RT-PCR revealed that untransducted EJ/T24 cells did not express LRIG1 or expression were below the limits of detection. After successful Ad-LRIG1 infection, LRIG1 mRNA and protein levels were remarkably up-regulated in EJ/T24 cells, as shown in Fig. 2.

Consistent with our previous studies, EGFR expression in $\mathrm{EJ} / \mathrm{T} 24$ cells was significantly attenuated $(\mathrm{p}<0.05)$ following infection with Ad-LRIG1. Additionally, nuclear pEGFR levels in EJ/T24 cells were lower in LRIG1-positive cells than in the blank control and green fluorescent protein (GFP) (Ad-GFP) groups $(\mathrm{p}<0.05)$. Moreover, in the blank control and Ad-GFP groups, CDDP treatment caused an increase in nuclear pEGFR expression. Conversely, nuclear pEGFR in the LRIG1-positive cells was not enhanced, even at the same concentration $(1 \mu \mathrm{g} / \mathrm{ml})$ and the same duration $(30 \mathrm{~min})$ of CDDP treatment (Fig. 3).

The proteasome inhibitor MG132 blocks the effect of LRIGI. MG132 was used to inhibit proteasome activity before EJ/

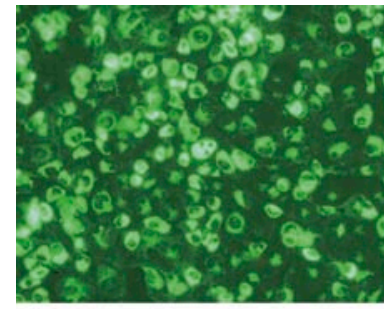

A

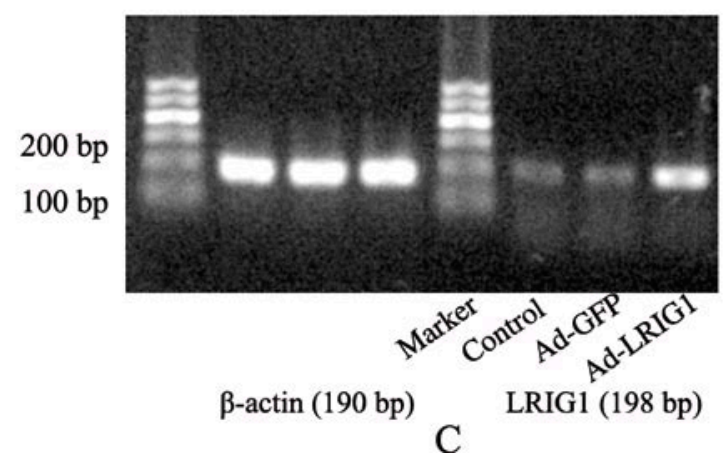

Figure 2. The expression of LRIG1 mRNA and protein in EJ/T24 cell lines infected with adenovirus vectors. (A) Cells infected with Ad-LRIG1; (B) LRIG1 protein expression in cells infected with Ad-LRIG1 compared to control groups; (C) LRIG1 mRNA expression in cells infected with Ad-LRIG1 compared to control groups.

T24 cells were infected by Ad-LRIG1. After pretreatment with MG132, there was no difference in CDDP-induced EGFR expression levels in all three groups (Control, DMSO and MG132, respectively) ( $>0.05)$, which means that EGFR expression levels were not down-regulated by LRIG1 after exposure to MG132. Furthermore, CDDP-induced nuclear pEGFR levels were more than 2-fold higher in MG132-treated cells than in untreated cells $(\mathrm{p}<0.05)$. In other words, CDDPinduced nuclear import of pEGFR was not blocked by LRIG1 in MG132-treated groups as much as in untreated groups (Fig. 4). Nevertheless, even taking the effects of MG132 into account, nuclear pEGFR levels in the LRIG1-positive groups were still lower than those of the LRIG1-negative groups. These results indicate that pEGFR nuclear import is limited by LRIG1 transduction even during treatment with a proteasome inhibitor.

LRIG1 facilitates CDDP-induced DNA damage and cellular apoptosis and reverses proliferation and invasion of EJ/T24 cells. To verify our hypothesis that LRIG1 might intensify CDDP-induced DNA damage by down-regulating nuclear import of EGFR, single-cell gel electrophoresis (SCGE) was used. Cells were treated as described in Fig. 5 and then were subjected to electrophoresis. Datum of tail-length and ratios were determined by Comet Assay Software Project (CASP) software. The Olive tail moment (OTM) value was used to evaluate the extent of DNA damage. LRIG1 combined CDDP treatment resulted in higher OTM values, indicating more severe DNA damage in LRIG1-positive cells than in LRIG1negative cells $(p<0.05)$. No statistically significant differences between CDDP and CDDP/Ad-GFP groups were observed $(p>0.05)$, though DNA damage in both groups was more severe than that of the blank control group $(\mathrm{p}<0.05)$. 
Control Ad-GFP Ad-LRIG1

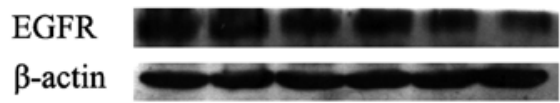

CDDP $0 \min 30 \mathrm{~min} 0 \mathrm{~min} 30 \mathrm{~min} 0 \mathrm{~min} 30 \mathrm{~min}$

A

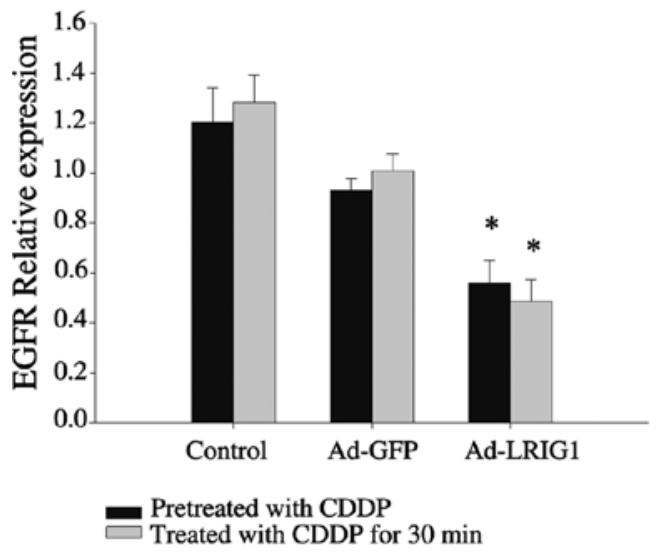

$\mathrm{C}$

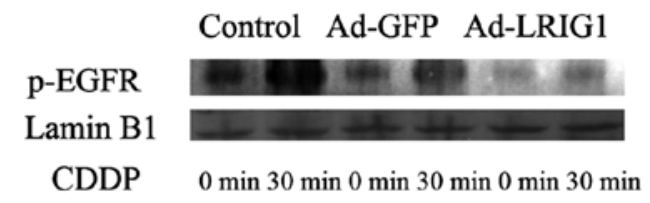

B

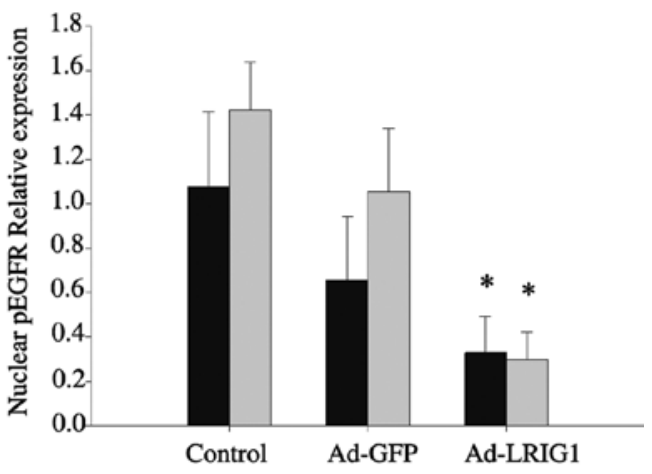

- Pretreated with CDDP

Treated with CDDP for $30 \mathrm{~min}$

D

Figure 3. LRIG1 attenuates EGFR and nuclear pEGFR expression in the EJ/T24 cell line. (A) EGFR expression levels in cells treated as shown above; (B) nuclear pEGFR expression levels in cells treated as shown above; (C) relative EGFR expression levels in cells treated as shown above; (D) relative nuclear pEGFR expression levels in cells treated as shown above ("p $<0.05)$.

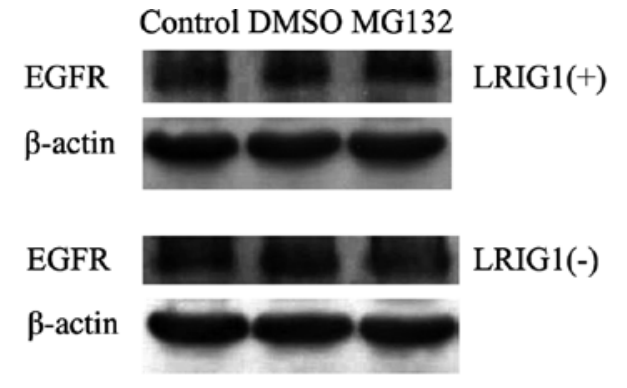

A

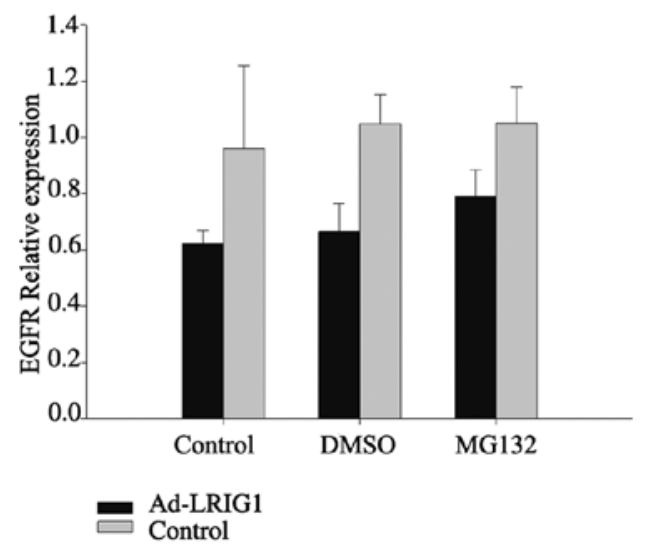

$\mathrm{C}$
Control DMSO MG132

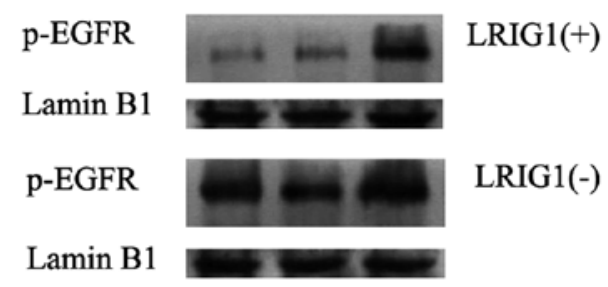

B

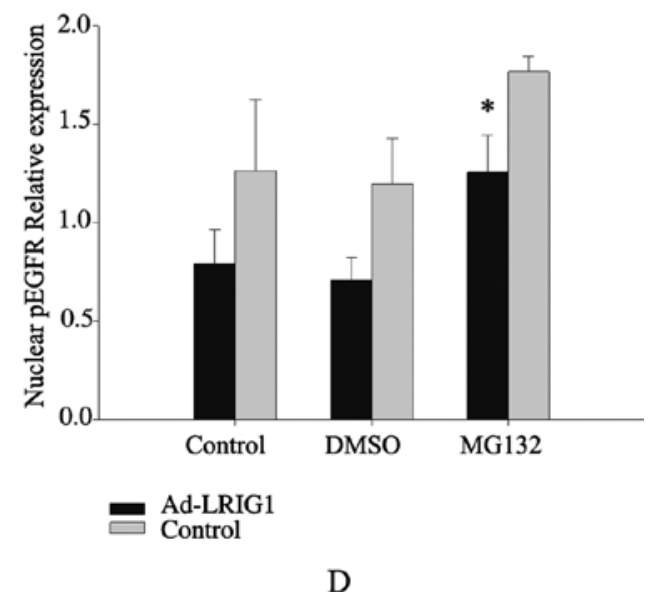

Figure 4. The proteasome inhibitor, MG132, blocks the effect of LRIG1 on EJ/T24 cells. (A) EGFR expression in cells cultured with $5 \mu \mathrm{M}$ MG132 for $12 \mathrm{~h}$ (DMEM and DMSO, as controls) prior to infection with Ad-LRIG1 or blank control (shown as LRIG1(+) and LRIG1(-), respectively). Each group was then treated with $1 \mu \mathrm{g} / \mathrm{ml}$ CDDP for $30 \mathrm{~min}$; (B) nuclear pEGFR expression in the groups as shown above; (C) relative EGFR expression in LRIG1(+) and LRIG1(-); (D) relative expression of nuclear pEGFR expression in LRIG1(+) and LRIG1(-) ( $(\mathrm{p}<0.05)$.

To determine whether LRIG1 and CDDP regulate the cell cycle, cells were stained with propidium iodide (PI) followed by fluorescence activated cell sorting (FACS). Compared to the control, more cells in the CDDP-treated groups were 

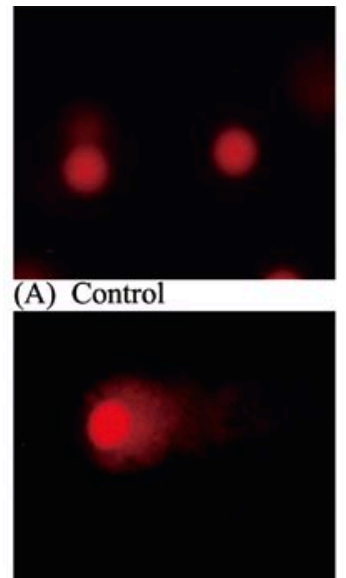

(C) CDDP/Ad-GFP

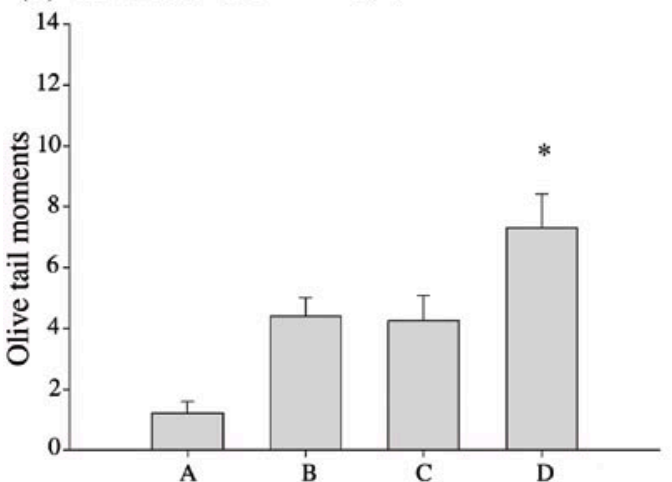

(E)

Figure 5. Evaluation of DNA damage in EJ/T24 cell lines by SCGE. (A) blank control (untreated with CDDP or LRIG1); (B) cells were treated with $1 \mu \mathrm{g} / \mathrm{ml}$ CDDP for $30 \mathrm{~min}$; (C) cells were infected with Ad-GFP for $48 \mathrm{~h}$ and treated with $1 \mu \mathrm{g} / \mathrm{ml}$ CDDP for $30 \mathrm{~min}$; (D) cells were infected with Ad-LRIG1 for $48 \mathrm{~h}$ and treated with $1 \mu \mathrm{g} / \mathrm{ml} \mathrm{CDDP}$ for $30 \mathrm{~min}$; (E) the four groups underwent SCGE, and OTM results are shown ( $(\mathrm{p}<0.05)$.

in the $\mathrm{G}_{0} / \mathrm{G}_{1}$ phase $[50.97 \pm 2.27 \%$ (Control) vs. $58.73 \pm 5.79 \%$ (CDDP), 63.34 $\pm 5.63 \%$ (CDDP/Ad-GFP)]. There was no significant difference between CDDP and CDDP/Ad-GFP cells ( $p>0.05$ ). In LRIG1-positive cells treated with CDDP, cells were obviously arrested in the $S$ phase $[31.4 \pm 3.21 \%$ (Control), $31.3 \pm 4.66 \%$ (CDDP), $25.81 \pm 6.79 \%$ (CDDP/Ad-GFP) vs. $46.40 \pm 1.81 \%$ (CDDP/Ad-LRIG1)], rather than other phases (Fig. 6). Moreover, the early apoptosis rates of LRIG1 positive groups were higher than the other three groups (data not shown).

Apoptosis was evaluated by Annexin V-APC/7-AAD double labeling for flow cytometry (FCM). LRIG1-transducted cells showed more early and late apoptosis than untransducted cells. [Early apoptotic cells, $5.18 \pm 3.36 \%$ (Control), $5.47 \pm 1.54 \%$ (CDDP), $9.33 \pm 1.63 \%$ (CDDP/Ad-GFP) vs $24.51 \pm 3.40 \%$ (CDDP/Ad-LRIG1) $(\mathrm{p}<0.05)$ and late apoptotic cells, $3.54 \pm 1.7 \%$ (Control), $5.83 \pm 1.53 \%$ (CDDP), $13.75 \pm 0.65 \%$ (CDDP/Ad-GFP) vs. $19.97 \pm 1.48 \%$ (CDDP/Ad-LRIG1) $(\mathrm{p}<0.05)]$ (Fig. 7).

Proliferating cell nuclear antigen (PCNA) expression was determined by immunocytochemistry, and PCNA-positive cells were defined as cells with staining in the nucleus. The ratio of PCNA-positive cells to total cells per visual field and total cell number in the visual fields were both assessed.

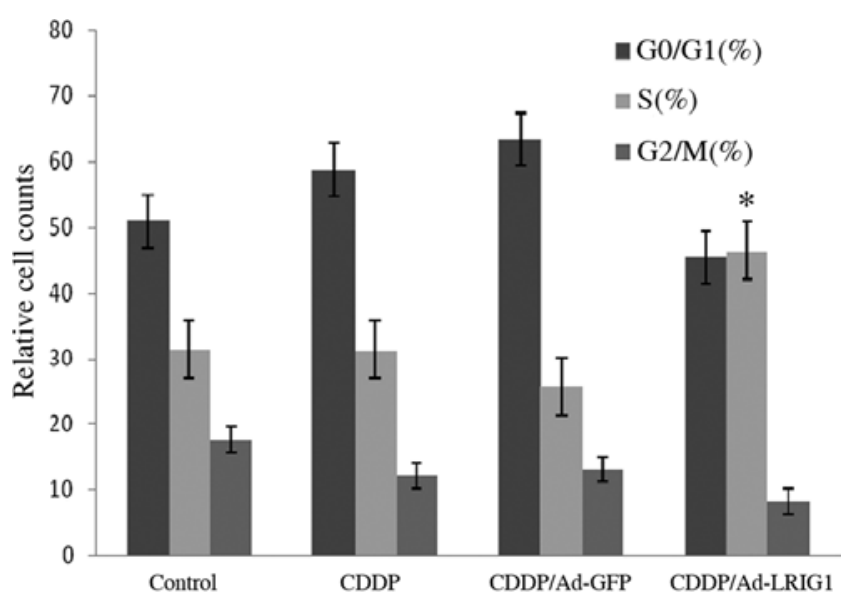

Figure 6. Effects of CDDP and LRIG1 on EJ/T24-cell cycle. Cells were treated according to groups (Control, CDDP, CDDP/Ad-GFP, CDDP/ Ad-LRIG1 as in Fig. 5) and analyzed by FCM. Percentage of cells in each phase of the cell cycle are displayed in the bar graph and presented as the mean \pm standard deviation $(* \mathrm{p}<0.05)$.

The CellQuest Research Software total number of cells was remarkably decreased in CDDP/Ad-LRIG1, and, conversely, the ratio of PCNA-positive cells to total cells per visual field was predominantly increased ( $\mathrm{p}<0.05)$ (Fig. 8).

Cell invasion was assessed in vitro by Matrigel invasion assay. The Matrigel matrix served as a reconstituted basement membrane, and the number of cells that migrated through the matrix was counted. LRIG1-positive cells treated with CDDP were significantly less invasive than LRIG1negative cells treated with CDDP $(\mathrm{p}<0.05)$, but there was no significant difference between Ad-GFP and untransducted cells ( $>>0.05$ ) (Fig. 9). These results demonstrate that the expression of LRIG1 was associated with a reduction of invasive ability.

\section{Discussion}

CDDP induces DNA damage by the formation of CDDP-DNA adducts. Most studies have focused on the effects and mechanisms of long-term, or late phase, CDDP-DNA damage. In contrast to the usual transient EGFR activation in response to oxidative stress, UV light and alkylating agents, CDDP induces consistent EGFR phosphorylation after DNA adduct formation, which lasts up to $24 \mathrm{~h}$ and involves src-family kinases (12). CDDP induces EGFR activation through phosphorylation of tyrosine 845 , which stabilizes the activation loop of EGFR, maintains the enzyme in the active state, provides a binding surface for protein substrates and leads to cell survival (13). Yet, until now, CDDP-induced immediate early DNA damage had not been fully understood.

To our knowledge, this is the first study that has investigated the immediate early phase of CDDP-induced DNA damage and activation and translocation of EGFR in a bladdercancer cell line. Similar results, have been found recently in the human bronchial carcinoma cell line, A549 (5). In the present study, nuclear import of phosphorylated EGFR was observed in the immediate early phase of DNA damage following CDDP exposure in EJ/T24 bladder cancer cells. 


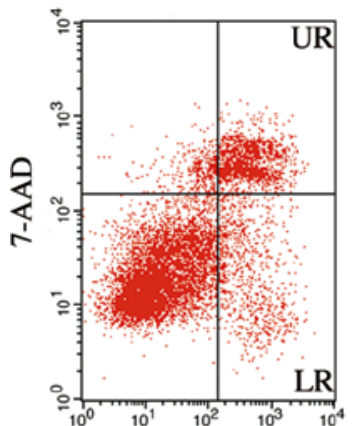

(A)

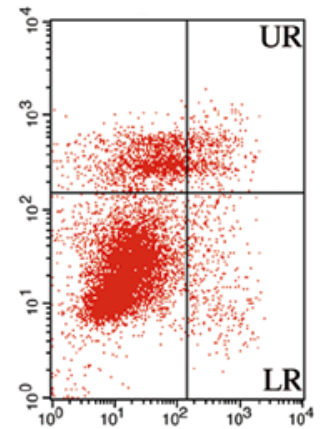

(B)

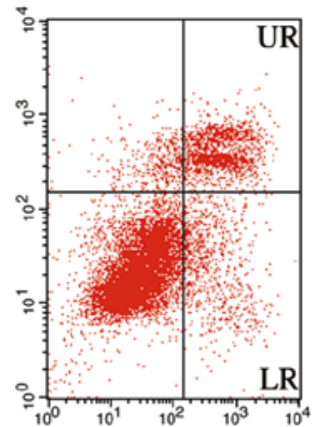

(C)

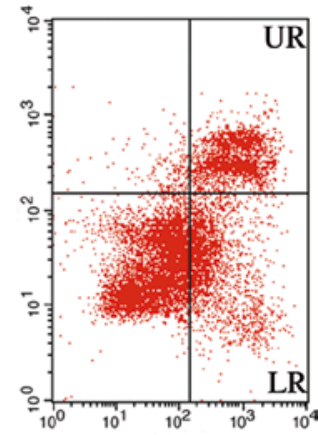

(D)

Control

CDDP

CDDP/Ad-LRIG1

Figure 7. Effects of CDDP and LRIG1 on EJ/T24-cell apoptosis. Cells were treated as described in Fig. 5 and analyzed by Annexin V-APC/7-AAD double

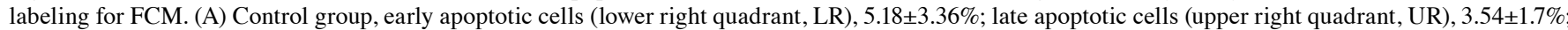
(B) CDDP group, early apoptotic cells, $5.47 \pm 1.54 \%$; late apoptotic cells, $5.83 \pm 1.53 \%$; (C) CDDP/Ad-GFP group, early apoptotic cells, $9.33 \pm 1.63 \%$; late apoptotic cells, $13.75 \pm 0.65 \%$; (D) CDDP/Ad-LRIG1 group, early apoptotic cells, $24.51 \pm 3.40 \%$; late apoptototic cells, $19.97 \pm 1.48 \%$.

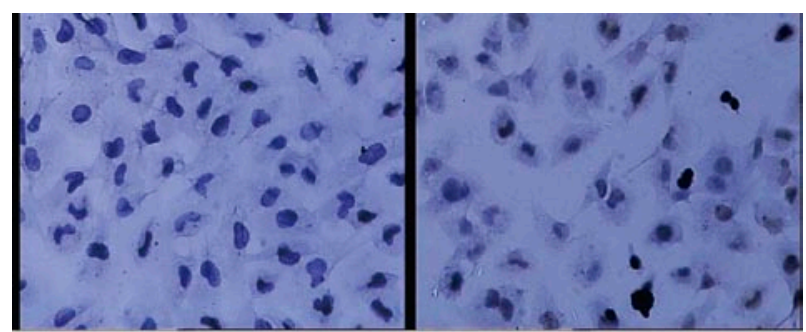

(A) Control

(B) CDDP

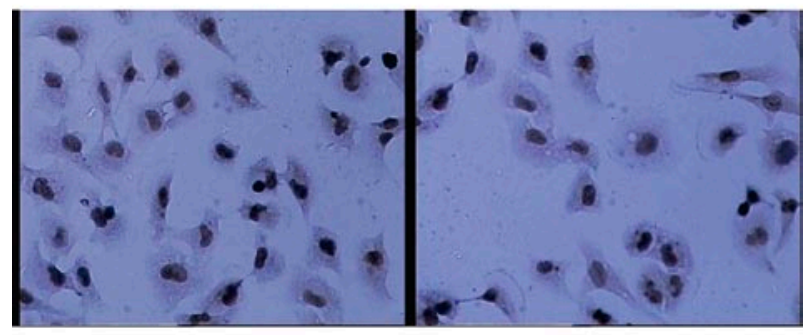

(C) CDDP/Ad-GFP

(D) CDDP/Ad-LRIG1

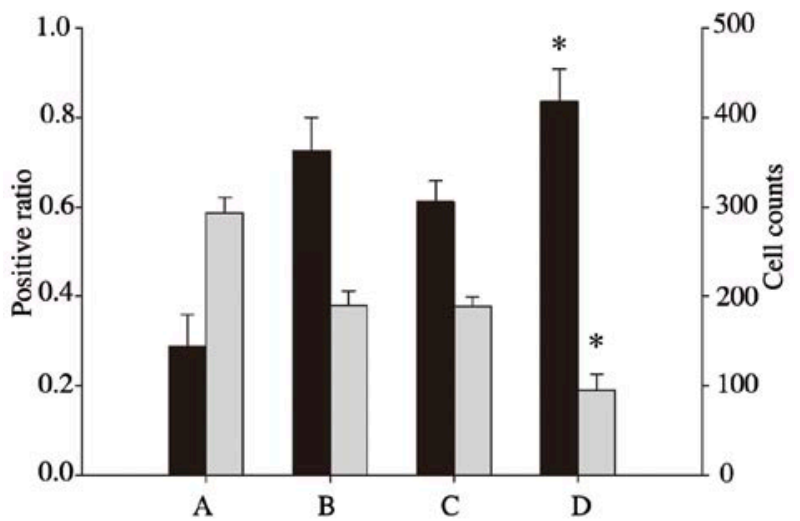

(E)

Figure 8. PCNA expression in EJ/T24 cells after LRIG1 transduction and exposure to CDDP. (A) PCNA expression in control cells; (B) PCNA expression in cells treated with CDDP; (C) PCNA expression in CDDP/Ad-GFP cells; (D) PCNA expression in CDDP/Ad-LRIG1 cells; (E) ratio of PCNApositive cells to total cells per visual field and total cell number in five visual fields are shown by bar graph ("p<0.05).

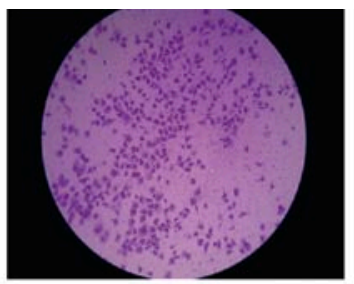

(A) Control

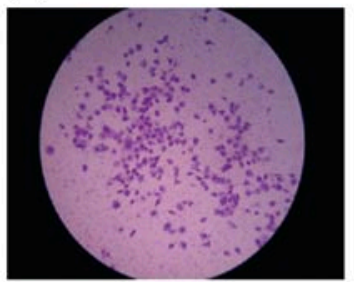

(C) CDDP/Ad-GFP

400

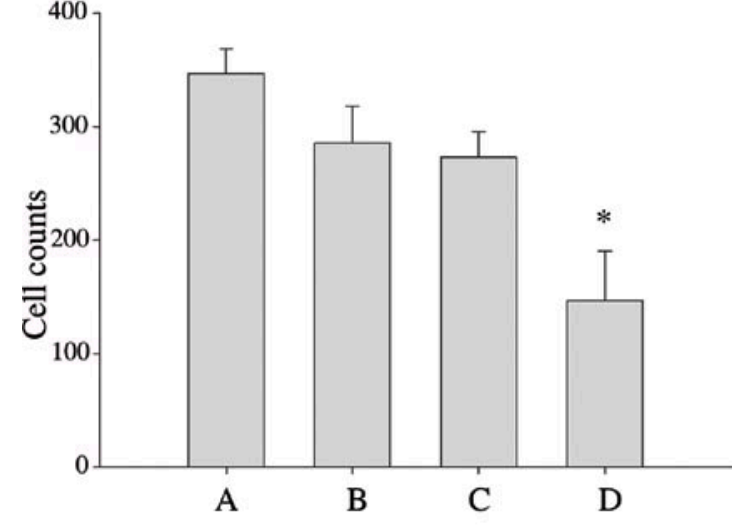

(E)

Figure 9. Effects of CDDP and LRIG1 on EJ/T24 cell invasion. Images were collected using a microscope at $\mathrm{x} 100$. Cells were treated as described in Fig. 5. (A) Control; (B) CDDP; (C) CDDP/Ad-GFP; (D) CDDP/Ad-LRIG1). (E) Cell counts are shown in the bar graph $\left({ }^{*} \mathrm{p}<0.05\right)$.

Therefore, CDDP, similar to ionizing radiation, induced EGFR activation and nuclear translocation. Dittmann et al observed that radiation-induced lipid peroxidation resulted in 
the generation of hydroxy-nonenal, which activated the redoxsensitive switch of src and caused a conformational alteration associated with increased kinase activity (14). Activated src phosphorylates EGFR at tyrosine 845 and caveolin 1 at Y14, which leads to internalization of EGFR into caveolae and transportation into the nucleus (15). As a result of EGFR internalization, nuclear pEGFR up-regulates DNA-PK activity and supports DNA-repair processes (16). Radiation induces DNA damage by causing double-strand breaks, while CDDP causes DNA damage by making CDDP-DNA adducts. However, as both have been shown to cause pEGFR internalization and evoke DNA repair, it was reasonable for us to believe that the mechanism mentioned above also applies to CDDP-induced nuclear import of EGFR.

LRIG1 was found to be absent in a high grade bladder cancer cell line. After LRIG1 was transducted into cells, EGFR expression was down-regulated and pEGFR nuclear import induced by CDDP oxidative stress was attenuated. It is currently accepted that LRIG1 likely destabilizes EGFR by facilitating the interaction of EGFR with protein degradation machinery. EGFR degradation has been found to depend on both proteasomes and lysosomes, and can be prevented with inhibitors of either. The $26 \mathrm{~S}$ proteasome controls cell cycle progression, cell survival, and cell death or degradation in response to stress stimuli by modulating the expression of specific signal transduction molecules. To further understand the mechanism by which LRIG1 down-regulates EGFR, cells were exposed to MG132, a specific and efficient 26S proteasome inhibitor, before LRIG1 transduction. EGFR expression levels were not changed by LRIG1 after the proteasome degradation pathway was blocked with MG132. This result confirms that LRIG1 regulates EGFR via the $26 \mathrm{~S}$ proteasome. Also, CDDP-induced nuclear import of pEGFR was not blocked by LRIG1 in MG132-treated cells as much as in untreated cells. Taking the effects of MG132 into account, nuclear pEGFR levels in LRIG1-transducted cells were still lower than those of untransducted cells. Taken together, these results indicate that down-regulation of pEGFR nuclear import by LRIG1 was limited by MG132, but not entirely ablated. Gur et al have shown that recruitment of $\mathrm{c}-\mathrm{Cbl}$ to the vicinity of EGFR caused ubiquitylation and subsequent degradation of LRIG1 and EGFR (8). In agreement with this, our present results indicate that LRIG1 reinforced ligandstimulated ubiquitination of EGFR and inhibited pEGFR translocation in a c-Cbl-dependent manner. We also found that accumulation of nuclear pEGFR after treatment with CDDP was lower in LRIG1-positive cells than LRIG1-negative cells, which implies that c-Cbl may not be the only pathway responsible for pEGFR translocation. Therefore, we suggest that LRIG1-mediated inhibition of CDDP-induced nuclear import of EGFR in the EJ/T24 cell line could have at least two causes: one is by accelerating ubiquitylation of EGFR, leading to its degradation by the $26 \mathrm{~S}$ proteasome, and another is by preventing phosphorylation of EGFR in response to oxidative stress and the subsequent internalization of EGFR into caveolae and transportation into nucleus (16).

However, the most intriguing results from our study was the detection of PCNA expression. As a classic biomarker for evaluating DNA duplication, PCNA expression starts and gradually increases during the $G_{1}$ phase, reaching its peak at the end of S phase (17). The overexpression of PCNA in malignant tissue is considered to be indicative of more aggressive and highly proliferative cancer cells (18). However, more recent studies have demonstrated that PCNA is engaged in DNA repair and is only a reflection of DNA damage after injury by various agents (19). Therefore, PCNA expression can be considered to be a mark of DNA duplication, whether or not injury is present $(19,20)$. Based on the evidence above, we believe that PCNA is up-regulated in response to DNA injury, and at the checkpoint from $G_{0} / G_{1}$ to $G_{2} / M$ phase, the gate keeper allows the cell cycle to progress if the injuries are repaired or directs cells toward apoptosis.

Flow cytometry revealed that, as a consequence of CDDP treatment, cells were accumulated in the $G_{1}$ phase. However, LRIG1-positive cells treated with CDDP were arrested in the $\mathrm{S}$ phase, which is known to be the checkpoint from $G_{0} / G_{1}$ to $G_{2} / M$ phase. In this study, the results from SCGE demonstrated that CDDP-induced DNA damage in LRIG1-positive cells might be correlated with blockade of pEGFR nuclear import and failure of DNA repair downstream. Thus, despite the increased PCNA staining in LRIG1-positive bladder cancer cells, CDDP-induced DNA damage might not be repaired as usual by up-regulation of sliding clamps, such as PCNA, and LRIG1-mediated suppression of pEGFR nuclear import seems to be involved. As a result, cells in CDDP/Ad-LRIG1 group were prone to apoptosis or cell death, which was obviously shown by PCNA-stained cell counts in Fig. 8 and flow cytometry in Fig. 7. This result implies that DNA injury, but not pEGFR nuclear import is necessary for PCNA up-regulation. However, nuclear pEGFR is engaged downstream of DNA repair involved in the PCNA feedback loop. As a consequence of DNA damage repair, cells treated with CDDP or other agents are able to escape from apoptosis via a multi-signaling network. The role of LRIG1 in this complicated network is still ambiguous and requires further study.

Advanced bladder cancer is characterized by invasive grow th that prevents total removal during tumor resection and initiates metastasis and recurrence. The Matrigel invasion assay suggested that the invasive ability of bladder cancer cells exposure to CDDP was attenuated by LRIG1, indicating that up-regulation of LRIG1 expression effectively inhibited the metastasis of EJ/T24 cells. This phenomenon might be related to synergism between CDDP-induced DNA damage and LRIG1-induced attenuation of EGFR expression.

In conclusion, our results contribute to a better understanding that increased LRIG1 leads to increased ubiquitylation and degradation of EGFR and might influence activation and translocation of EGFR, which plays an important role in preventing DNA damage by oxidative stress. Strategies based on down-regulating EGFR expression with LRIG1 might comprise a novel therapeutic approach to improve the response of bladder cancer to chemotherapy. Studies in vivo to corroborate our findings in vitro are already underway in our laboratory.

\section{Acknowledgements}

This study was supported by Chinese National Nature and Science fund (No. 30500521 and 30872653). We are grateful to 
Professor Håkan Hedman and Dr Jonas Nilsson for providing the pLRIG1-GFP plasmid, and Dr Zhulin Liang for providing the cell line. Baofeng Wang is acknowledged for his kindly assistance with technical support.

\section{References}

1. Byun SS, Kim SW, Choi H, Lee C and Lee E: Augmentation of cisplatin sensitivity in cisplatin-resistant human bladder cancer cells by modulating glutathione concentrations and glutathionerelated enzyme activities. BJU Int 95: 1086-1090, 2005.

2. Perry JJ and Muss HB: Management of disseminated disease in the patient with bladder cancer. Urol Clin North Am 21: 661-672, 1994.

3. Brozovic A and Osmak M: Activation of mitogen-activated protein kinases by cisplatin and their role in cisplatin-resistance. Cancer Lett 251: 1-16, 2007.

4. Lo HW and Hung MC: Nuclear EGFR signalling network in cancers: linking EGFR pathway to cell cycle progression, nitric oxide pathway and patient survival. Br J Cancer 94: 184-188, 2006.

5. Dittmann K, Mayer C, Fehrenbacher B, et al: Radiation-induced epidermal growth factor receptor nuclear import is linked to activation of DNA-dependent protein kinase. J Biol Chem 280: 31182-31189, 2005.

6. Dittmann K, Mayer C and Rodemann HP: Inhibition of radiationinduced EGFR nuclear import by C225(Cetuximab) suppresses DNA-PK activity. Radiother Oncol 76: 157-161, 2005.

7. Dittmann K, Mayer C and Rodemann HP: Nuclear EGFR as novel therapeutic target: insights into nuclear translocation and function. Strahlenther Onkol 186: 1-6, 2010.

8. Gur G, Rubin C, Katz M, et al: LRIG1 restricts growth factor signaling by enhancing receptor ubiquitylation and degradation. EMBO J 23: 3270-3281, 2004.

9. Yang WM, Yan ZJ, Ye ZQ and Guo DS: LRIG1, a candidate tumour-suppressor gene in human bladder cancer cell line BIU87. BJU Int 98: 898-902, 2006.
10. Olive PL and Banáth JP: The comet assay: a method to measure DNA damage in individual cells. Nat Protoc 1: 23-29, 2006.

11. Końca K, Lankoff A, Banasik A, et al: A cross-platform public domain PC image-analysis program for the comet assay. Mutat Res 534: 15-20, 2003.

12. Benhar M, Engelberg D and Levitzki A: Cisplatin-induced activation of the EGF receptor. Oncogene 21: 8723-8731, 2002.

13. Tice DA, Biscardi JS, Nickles AL and Parsonss SJ: Mechanism of biological synergy between cellular Src and epidermal growth factor receptor. Proc Natl Acad Sci USA 96: 1415-1420, 1999.

14. Dittmann K, Mayer C, Kehlbach R, Rothmund MC and Rodemann HP: Radiation-induced lipid peroxidation activates src kinase and triggers nuclear EGFR transport. Radiother Oncol 92: 379-382, 2009.

15. Dittmann K, Mayer C, Kehlbach R and Rodemann HP: Radiation-induced caveolin-1 associated EGFR internalization is linked with nuclear EGFR transport and activation of DNA-PK. Mol Cancer 7: 69, 2008.

16. Chen DJ and Nirodi CS: The epidermal growth factor receptor: a role in repair of radiation-induced DNA damage. Clin Cancer Res 13: 6555-6560, 2007.

17. Kurki P, Vanderlaan M, Dolbeare F, Gray J and Tan EM: Expression of proliferating cell nuclear antigen (PCNA)/cyclin during the cell cycle. Exp Cell Res 166: 209-219, 1986.

18. El-kott AF, El-baz MA and Mokhtar AA: Proliferating cell nuclear antigen (PCNA) overexpression and microvessel density predict survival in the urinary bladder carcinoma. Int Urol Nephrol 38: 237-242, 2006.

19. Negishi M, Chiba T, Saraya A, Miyagi S and Iwama A: Dmap1 plays an essential role in the maintenance of genome integrity through the DNA repair process. Genes Cells 14: 1347-1357, 2009.

20. Politi EN, Lazaris AC, Lambropoulou S, Alexopoulou D, Kyriakidou V and Koutselini H: Epidermal growth factor receptor and proliferating cell nuclear antigen expression in urine ThinPrep specimens. Cytopathology 16: 303-308, 2005. 